\title{
ESG AND CORPORATE BAILOUTS: SEPTEMBER 2020 SURVEY
}

\author{
EMANUELE COLONNELLI* AND NIELS JOACHIM GORMSEN ${ }^{\dagger}$
}

\section{InTRODUCTION}

This project aims to understand whether and how perceptions about corporate ESG affect support for corporate bailouts and related policies. We do so by means of an experimental survey where we randomly vary individual perceptions of ESG practices of large corporations (through original short animated videos).

Note: this pre-analysis plan builds on the original pre-analysis plan AEARCTR-0005806, which contains the design of the original survey. This September 2020 experimental survey aims at (a) replicating the main results of our original study at a different point in time (where the COVID and bailout discussion are less intertwined than at its peak when we conducted the original survey) and (b) obtaining additional outcome measures, namely costly behavioral actions and open-ended questions.

\section{DATA}

We aim to collect survey data on a sample of 2,000 individuals that is representative of the US working-age population. The precise number may slightly differ depending on the ability of our partner Dynata to recruit new respondents and to guarantee a representative sample on the dimensions of gender, age, education, income, employment status, race, and political orientation.

The survey contains four main sections:

(1) Socio-demographic questions on gender, age, income, ethnicity, education, employment, and political views.

(2) Perceptions about corporate ESG policies

(3) Support for corporate bailouts

(4) Open-ended questions and respondents' views about the survey.

As explained below, the surveys also include videos. To check understanding of the videos, we introduce basic test questions during and after the videos. Respondents are required to answer correctly to continue the survey.

Note: compared to our May 2020 survey, we added several questions in sections 3 and 4 above. Hence, to keep the survey relatively short, we eliminated a few questions from the earlier survey.

\section{Experimental Design}

3.1. Randomization. We introduce only one source of variation in this September survey. Specifically, we introduce a video (which varies depending on the control/treatment arm) that is shown after Section 1 of the survey. This treatment is aimed at randomly varying the respondents' perceptions about how bad corporations are (from an ESG standpoint).

${ }^{\star}$ Booth School of Business, University of Chicago. emanuele.colonnelli@chicagobooth.edu.

${ }^{\dagger}$ Booth School of Business, University of Chicago. niels.gormsen@chicagobooth.edu. 
Specifically, we split the sample into 2 groups $(\mathrm{N}=$ target sample size):

- Control $[\mathrm{N}=1,000]$ : the video explains key concepts such as corporate bailouts and shareholders vs stakeholders. This survey is akin to the "Control Pre" survey we had in the May experiment.

- ESG-Neg $[\mathrm{N}=1,000]$ : the video explains key concepts such as corporate bailouts and shareholders vs stakeholders, plus scenes providing examples of corporate ESG policies when corporations primarily care about shareholders. This survey is akin to the "ESGNeg Pre" survey we had in the May experiment.

The randomization is done by our research partner Dynata.

Note: Compared to the May 2020 experiment, we decided to drop the second layer of randomization and the ESG-Pos and Economy treatments. This is driven primarily by financial consideration, namely the budget at our disposal for this second experiment, which required us to limit the number of treatment arms. Second, we opted for this streamlined experiment due to the fact that the economics of our analysis are well summarized by the Control vs ESG-Neg comparison only, as it was stated also in the previous pre-analysis plan.

3.2. Analysis. Our main analysis of the experimental results will consist of running regressions of the form:

$$
Y_{i}=\beta_{0}+\beta_{1} T_{i}+X_{s}^{\prime} \gamma+\epsilon_{i}
$$

where $Y_{i}$ is the given outcome variable for individual $i$ measured in our survey, and $T_{i}$ is an indicator equal to one if individual $i$ received the treatment. $X_{s}$ is a vector of control variables at the individual level such as socio-demographic status, and $\epsilon_{i}$ is the error term. As in our May 2020 survey, we will measure ESG perceptions primarily through the difference between what respondents think corporations do and what they think corporations should do (as a measure of how unsatisfied they are with large corporations ESG policies).

Our main outcomes are the questions on support for corporate bailouts. We ask the same self-reported question we asked in May 2020 (support for corporate bailouts from 0 to 10). Importantly, we added three more questions aimed at measuring "support for bailouts", involving a real (costly) action from the respondent. First, we ask whether they would like to donate part of their winnings (via a lottery) to a non-profit organization (Business Roundtable) that represents executives of large American corporations (as a way to capture not just the support for a specific policy, like bailouts, but more broadly a change in attitude towards supporting large corporations). Second, we ask whether they would like to sign a petition that asks the House of Representatives and the Senate to provide additional bailout money to large corporations. Third, we ask them to give us the OK to send a message to Senators of their choice, with their name in the signature, asking to support or oppose bailouts (we think our treatment will make it less likely to sign the support email, and more likely to sign the oppose email).

As in our May 2020 survey, we are also interested in additional analyses aimed at estimating heterogeneous effects of our treatments. In particular, we plan to study differences across socio-demographic groups, with an emphasis on political views of individual respondents.

Finally, we ask three incentivized open-ended questions, asking respondents why they have certain views about corporations, why they decided or not to undertake behavioral costly actions, and what they think is the purpose of a corporation. We ask these questions at the end of the surveys, so as not to contaminate the analysis above. We ask these open ended questions to further investigate the mechanisms driving the effects we may find. Specifically, we think our treatment may lead individuals to support large corporations, and policies that are friendly to large corporations, less because of their insufficient efforts to be good corporate 
citizens. Similarly, we think the decreased support towards large corporations may also be affected by lower trust in both corporations and government institutions, which may reduce the support for government policies like bailouts, or the willingness of individuals to take costly behavioral actions like signing a petition or contacting Senators. We plan to analyze the openended text primarily through unsupervised machine learning algorithm like LDA, following similar methodologies as in Stantcheva (2020, "Understanding Economic Policies: What Do People Know and Learn?") and in Bursztyn et al (2020, "I Have Nothing Against Them, But..."). 